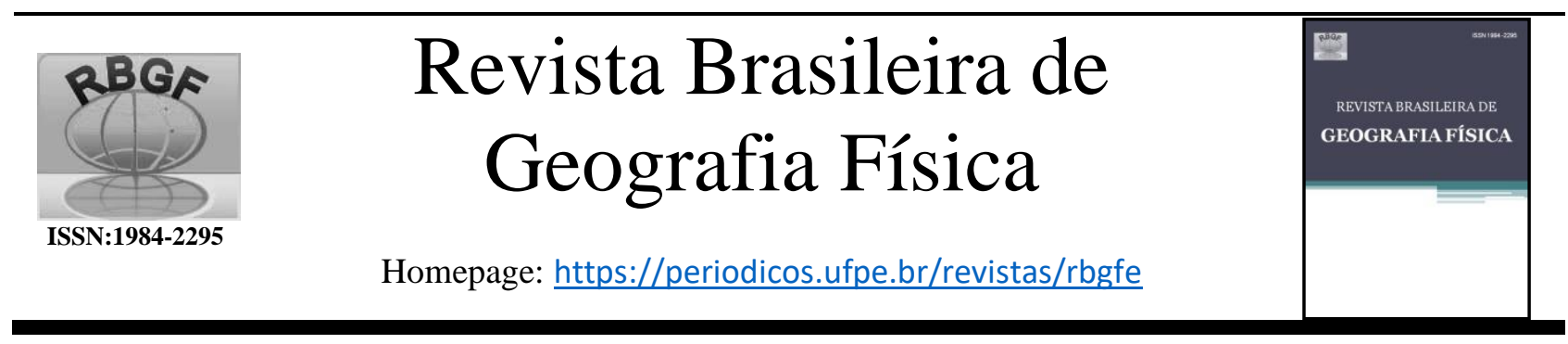

\title{
Avaliação da temperatura da superfície no Pantanal Mato-grossense por Sensoriamento Remoto
}

\author{
Otávio Rodrigues Mendes ${ }^{1}$, Victor Hugo de Morais Danelichen ${ }^{2}$, Osvaldo Alves Pereira ${ }^{3}$
}

\begin{abstract}
${ }^{1}$ Graduando em Engenharia Ambiental, Universidade de Cuiabá, Campus Barão, 222, CEP 78005-300, Cuiabá, Mato Grosso. (65) 3363-1729. otaviodnf2@ hotmail.com. ${ }^{2}$ Professor doutor e Pesquisador, Mestrado em Ciências Ambientais, Universidade de Cuiabá, Campus Barão, 222, CEP 78005-300, Cuiabá, Mato Grosso. (65) 3363-1729. danelichen@ fisica.ufmt.br (autor correspondente). ${ }^{3}$ Professor doutor e Pesquisador, Mestrado em Ciências Ambientais, Universidade de Cuiabá, Campus Barão, 222, CEP 78005-300, Cuiabá, Mato Grosso. (65) $3363-1729$. osvaldoalvesfu@gmail.com.
\end{abstract}

Artigo recebido em 23/07/2019 e aceite em 20/11/2019

\section{R E S U M O}

Devido sua grande importância, o Pantanal é objeto de estudos de trabalhos que visam conhecer e proteger sua biodiversidade. Como instrumento indispensável, o Sensoriamento Remoto surge como uma ferramenta ideal para o mapeamento, identificação de queimadas, desmatamento e estudos climáticos. Dentre as variáveis estimadas pelos sensores orbitais, a temperatura da superfície é a menos explorada. Estudos que são conduzidos para validação da temperatura da superfície por meio de sensores orbitais e de estações meteorológicas no bioma Pantanal são escassos. Diante disso, este trabalho visou analisar a dinâmica da temperatura da superfície em uma unidade de conservação no norte do Pantanal a partir de imagens adquiridas do satélite Landsat 8. O estudo foi realizado em uma torre micrometeorológica, localizada entre os municípios de Cuiabá e Santo Antônio de Leverger - MT, situada na encosta de uma unidade de conservação, denominada Monumento Natural Morro de Santo Antônio. Para validação da temperatura da superfície, foram adquiridas imagens do satélite Landsat 8 fornecidos pela USGS. Resultados encontrados nesse trabalho demonstraram o uso das bandas termais do Landsat 8 no norte do Pantanal mato-grossense na discriminação de diferentes alvos na superfície. A análise parcial de cada banda do sensor TIRS demonstrou valores mais elevados na banda 10. A análise estatística dos dados indica que as maiores correlações da temperatura medida na torre meteorológica foram constatadas com a média das bandas termais do satélite Landsat 8. A análise de variância demonstrou que houve diferença significativa entre as médias das temperaturas medida e estimada.

Palavras-chaves: banda termal, sensor orbital, Pantanal.

\section{Surface temperature evaluation in the Pantanal Mato Grosso by Remote}

\section{Sensing}

\begin{abstract}
A B S T R A C T
Due to its great importance, the Pantanal is the object of studies that aim to know and protect its biodiversity. As an indispensable tool, Remote Sensing emerges as an ideal tool for the mapping, identification of fires, deforestation and climatic studies. Among the variables estimated by the orbital sensors, the surface temperature is the least explored. Studies that are conducted for surface temperature validation by orbital sensors and meteorological stations in the Pantanal biome are scarce. The study was carried out in a micrometeorological tower, located between the municipalities of Cuiabá and Santo Antônio, located on the slope of a conservation unit, called Natural Monument Morro de Santo Antônio. To validate the surface temperature, images of the Landsat 8 satellite provided by the USGS were acquired. Results obtained in this work demonstrated the use of the Landsat 8 thermal bands in the northern Pantanal of Mato Grosso in the discrimination of different surface targets. The partial analysis of each TIRS sensor band showed higher values in the 10 band. Statistical analysis of the data indicates that the highest correlations of the temperature measured in the meteorological tower were verified with the average of the thermal bands of the satellite Landsat 8 . The analysis of variance showed that there was a significant difference between the measured and estimated temperatures.
\end{abstract}

Keywords: thermal band, orbital sensor, Pantanal. 


\section{Introdução}

O bioma Pantanal constitui $150.355 \mathrm{~km}^{2}$ em solo brasileiro, estendendo pelo Paraguai e Bolívia, onde é conhecido como Chaco (Danelichen et al., 2016, Vigano et al., 2018). Este ainda representa $83,14 \%$ da sua vegetação nativa segundo o Projeto de Monitoramento do Desmatamento nos Biomas Brasileiros por Satélite (Andrade et al, 2017, Vilani et al., 2018).

Devido sua grande importância nacional e internacionalmente, o Pantanal, como os demais biomas, é objeto de estudos de trabalhos que visam conhecer e proteger sua biodiversidade (Dallacort et al., 2014, Aquino et al., 2017, Miranda, et al., 2018, Vigano et al., 2018).

Como instrumento indispensável a esses estudos, o Sensoriamento Remoto surge como uma ferramenta ideal para o mapeamento de áreas, identificação de queimadas, desmatamento, estudos climáticos, ademais, apresenta custo muito menor que a instalação, manutenção e obtenção de dados de uma estação meteorológica (Souza et al., 2014, Silva Junior et al, 2018, Velasque et al., 2018).

Além disso, o uso de geotecnologias é indispensável no tratamento e obtenção de imagens da superfície terrestre adquiridas por sensores orbitais, por meio das interações da radiação eletromagnética com alvos ali encontrados (Santos et al., 2015, Marques et al., 2017, Takatori et al, 2017).

Com o intuito de mapear os recursos naturais ao redor do globo, foi lançado pela NASA (National Aeronautics and Space Administration) o programa espacial Landsat. Seu primeiro satélite foi o ERTS - 1 lançado em 1972. Depois vieram o Landsat 2, 3, 4 e suas sequências 5 e 7 (Zhou et al., 2016, Knox et al., 2017). Em fevereiro de 2013 entrou em operação o satélite Landsat 8 (Pires \& Junior, 2015, Vermote et al., 2016). Esses satélites têm como uma de suas finalidades medir e analisar a temperatura da superfície através de sensores imageadores que atuam de acordo com as faixas do espectro eletromagnético (Santos et al., 2017, Takatori et al, 2017, Souza \& Danelichen, 2018).

Uma das novidades do Landsat 8 são os dois novos sensores imageadores, o Operacional Terra Imager (OLI) e Thermal Infrared Sensor (TIRS). O OLI vem com as bandas multiespectrais de 1 a 7 e a banda 9 . Também, conta com a banda 8 pancromática com resolução espacial de 15 metros. Já o TIRS conta com as bandas térmicas 10 e 11 que fornecem temperaturas de superfície mais precisas (Knight \& Kvarab, 2014., Zhou et al., 2016, Souza et al, 2018).

Dentre as variáveis estimadas pelos sensores orbitais, a temperatura da superfície é a menos explorada, tendo em vista a grande dificuldade de validar com dados terrenos estimados, e muitas vezes não corresponder com a temperatura do ar, pois a medida térmica obtida pela banda termal desses sensores, diz respeito a temperatura radiométrica da superfície desprezando os fluxos horizontais da biosfera (Strassburge et al., 2011, Alves et al., 2017).

A temperatura de superfície diz respeito ao fluxo de calor dado em função da energia que chega e sai do corpo, sendo de suma importância para o entendimento das interações entre a superfície terrestre e a atmosfera (Zibognon et al., 2002, Rosas et al., 2017, Lima et al, 2018).

Dessa forma, estudos são conduzidos para que se possa validar e comparar a temperatura da superfície por meio de sensores orbitais e de estações meteorológicas que possam fornecer dados da temperatura local (Anjos, 2013; Silva et al., 2014, Masiello et al., 2015, CristóbaL et al., 2018), no entanto, estudos desse tipo no bioma Pantanal é escasso.

Diante disso, o presente artigo visa analisar a dinâmica da temperatura da superfície em uma unidade de conservação no norte do Pantanal a partir de imagens adquiridas do sensor TIRS do satélite Landsat 8.

\section{Material e métodos}

\section{Área de estudo}

O estudo foi realizado com dados de temperatura do ar obtidos por dois termohigrômetros (Campbell HC2S3-QD) instalados a 2 e $3 \mathrm{~m}$ de altura em uma torre micrometeorológica, localizada entre os municípios de Cuiabá e Santo Antônio de Leverger (Figura 1), essa estação se encontra na encosta de uma unidade de conservação, denominada Monumento Natural Morro de Santo Antônio (Lei $\left.\mathrm{n}^{\circ} 8.504 / 2006\right)$, de coordenadas $15^{\circ} 45^{\prime} 45^{\prime \prime} \mathrm{S}$ e $56^{\circ} 03^{\prime} 57^{\prime} \mathrm{O}$ e altitude $202 \mathrm{~m}$ (Danelichen et al. 2014).

Segundo a classificação de Köppen o clima predominante no Pantanal é o Aw, clima tropical, com inverno seco (de maio a outubro). Durante o verão, ocorre a estação chuvosa, que vai de novembro a abril. As precipitações anuais variam entre $800 \mathrm{~mm}$ a $1.200 \mathrm{~mm}$ anuais. 


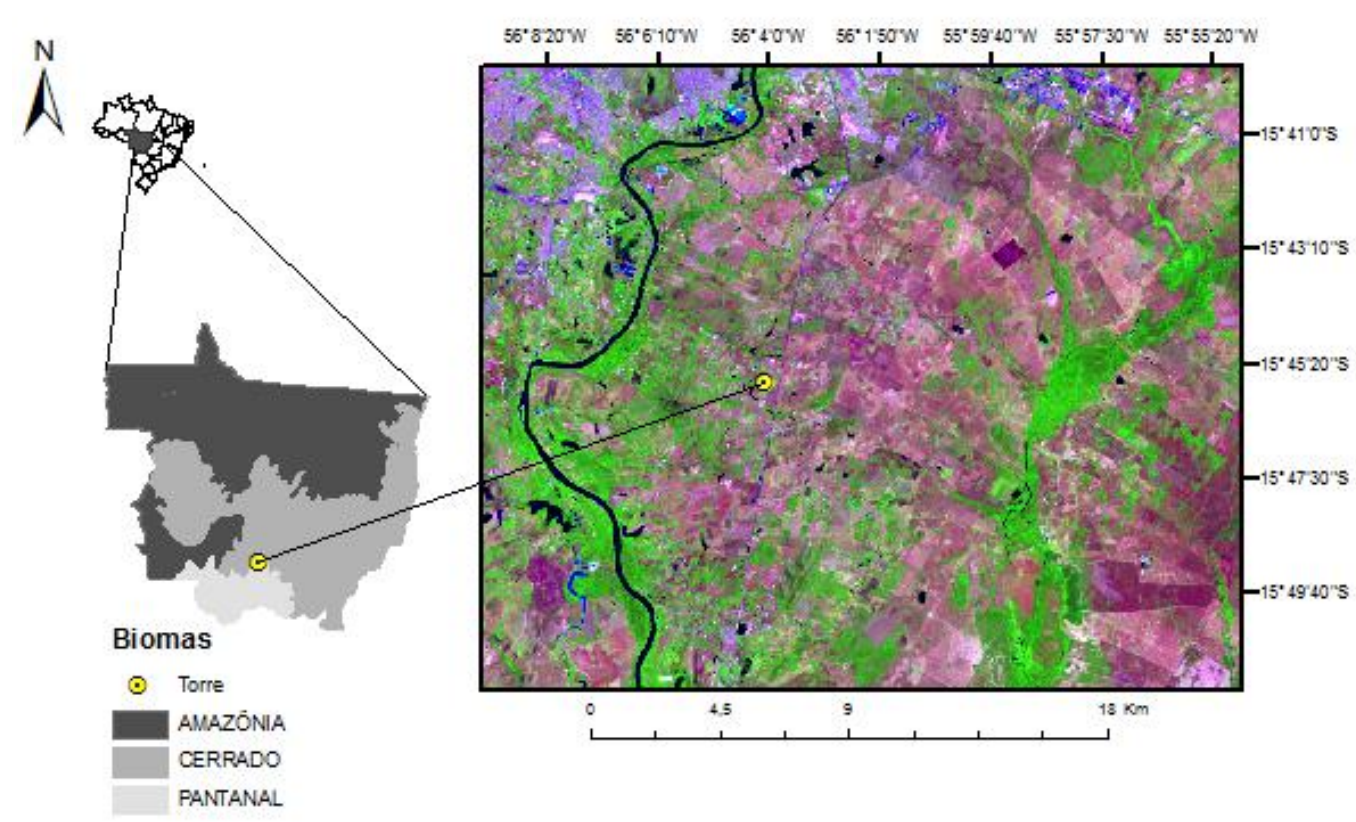

Figura 1. localização da área de estudo.

\section{Dados de Sensores Orbitais}

Para comparativo e validação da temperatura da superfície, foram adquiridas imagens do satélite Landsat 8 fornecidos pela USGS (United States Geological Survey) no endereço (https://www.usgs.gov). As imagens correspondem as bandas termais 10 e 11 , sendo alusivas aos dias 24/07/2016, 10/08/2016, 26/08/2016, 11/08/2016 e 27/08/2016. Escolheu-se essas datas devida a nenhum recobrimento de nuvens sobre a área de estudo. O horário registrado da passagem do satélite foi às 10:00hs da manhã (horário de Brasília).

Para auxilio no uso dessas imagens, utilizou-se o software ArcGis, para cômputo da média de temperatura. Com o auxílio de um algoritmo desenvolvido com a linguagem de programação Python, foi possível adquirir a temperatura média do pixel correspondente as coordenadas da torre.

\section{Obtenção da temperatura aparente da superfície}

Para o computo da temperatura da superfície das bandas 10 e 11 do Landsat 8 foi necessário primeiro fazer a conversão dos níveis de cinza das bandas em radiância espectral (TOA) por meio da equação (Barsi et al., 2014):

$$
L_{\lambda}=M_{L} Q_{c a l}+A_{L}
$$

onde $\mathrm{L}_{\lambda}$ é a radiância espectral $\left(\mathrm{W} / \mathrm{m}^{2} \operatorname{sr} \mu \mathrm{m}\right), \mathrm{M}_{\mathrm{L}}$ é o Fator multiplicativo de redimensionamento da

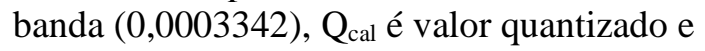

calibrado do pixel em nível de cinza (DN) e $\mathrm{A}_{\mathrm{L}}$ é o fator aditivo de redimensionamento da banda (0.1000).

Então fez-se a conversão da radiância espectral para temperatura da superfície através da equação:

$$
T=\frac{K_{2}}{\ln \left(\frac{K_{1}}{L_{\lambda}}+1\right)}
$$

onde $\mathrm{T}$ é Temperatura sem correção atmosférica (Kelvin), $\mathrm{K}_{1}$ é a constante de calibração 1 (774,89 para banda 10 e $480,89 \mathrm{~W} / \mathrm{m}^{2} \mathrm{sr} \mu \mathrm{m}$ para a banda $11), K_{2}$ é a constante de calibração $2(1321,08 \mathrm{~K}$ para banda 10 e 1201,14 K para banda 11). Posteriormente as temperaturas da superfície foram convertidas para graus Celsius $\left({ }^{\circ} \mathrm{C}\right)$ com a subtração do valor de 273,15 K.

\section{Análise estatística}

Os dados da temperatura do ar medida na torre e estimada pelo satélite Landsat 8 apresentaram distribuição normal pelos testes de Shapiro-Wilk, Anderson-Darling e KolmogorovSmirnov. A análise de variação foi realizada para verificar se as variáveis apresentaram diferença significativa entre si $(p$-valor $<0,05)$.

\section{Resultados e discussão}

A temperatura do ar variou de $25,4{ }^{\circ} \mathrm{C}$ a $38,7{ }^{\circ} \mathrm{C}$ ao longo dos meses analisados que compreende a estação seca (julho a setembro) apresentando uma média de $34,1^{\circ} \mathrm{C}$ (Figura 2). A 
mais alta temperatura encontrada no mês de agosto é condizente com o mês que mais sofre déficit hídrico nessa porção do bioma (Danelichen et al., 2013). Enquanto que, a temperatura da superfície estimada pelo satélite Landsat 8 apresentou comportamento semelhante a temperatura do ar medida na torre, variando de $22{ }^{\circ} \mathrm{C}$ a $29^{\circ} \mathrm{C}$, com média de $26,6{ }^{\circ} \mathrm{C}$ (Figura 2). Observa-se que há uma defasagem notável entre as médias das duas temperaturas, de aproximadamente $7,5^{\circ} \mathrm{C}$, que corresponde a $22 \%$ a menos, quando estimada pelo sensor TIRS do satélite Landsat 8 (Figura 2).

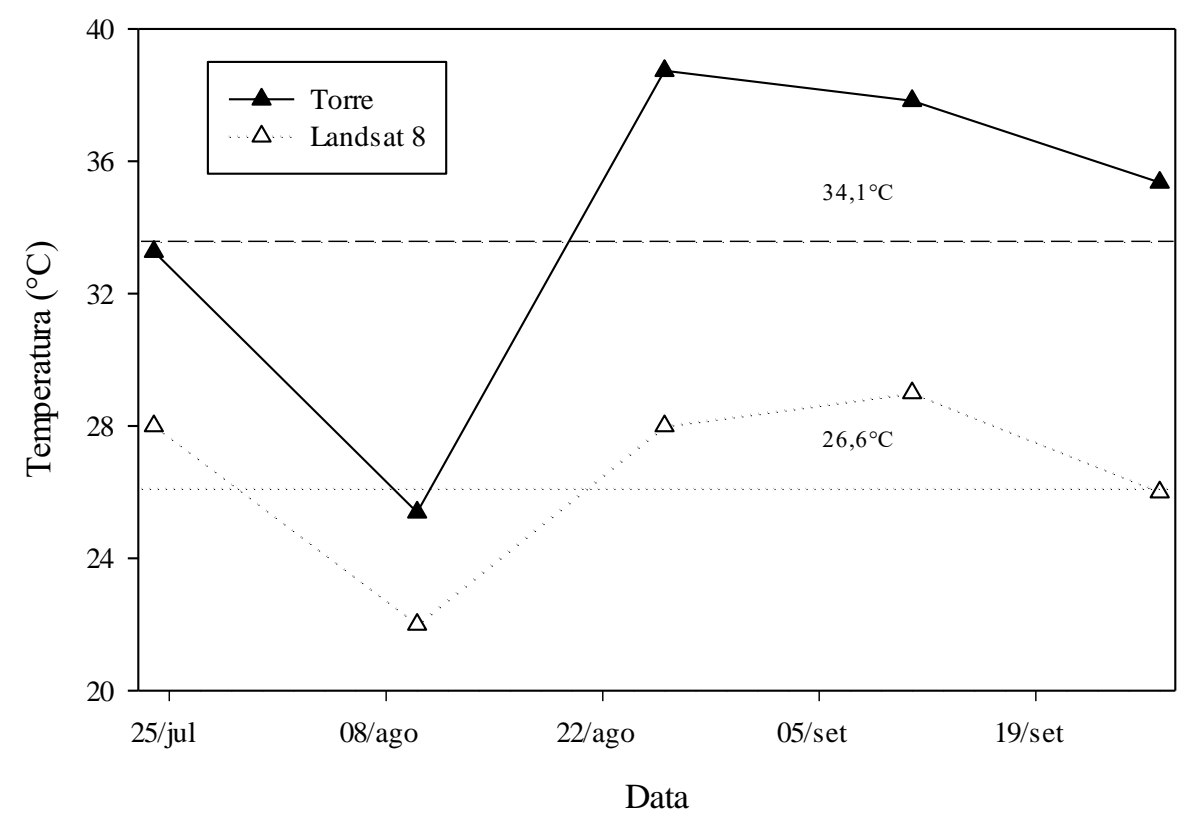

Figura 2. Temperatura do ar medido na torre e temperatura da superfície estimada pelas médias das bandas termais do satélite Landsat 8 na área de estudo.

Observa-se que os valores de temperatura da superfície estimada pelo satélite Landsat 8 do alvo (torre micrometeorológica) apresentaram pouca variação entre as duas bandas termais, aproximadamente $1,8^{\circ} \mathrm{C}$ (Tabela 1 ).

A banda termal 10 apresentou uma média de $27,8^{\circ} \mathrm{C}$ no pixel da torre em todo período analisado, um desvio padrão de 2,6, com valores mínimos no mês de agosto e máximos em setembro (Tabela 1). Enquanto, que a banda termal 11 apresentou média de $26^{\circ} \mathrm{C}$, desvio padrão de 2,3 , com valores mínimos e máximos nos mesmos meses da banda 10 (Tabela 1).

Tabela 1. Dados de Temperatura da torre e do satélite Landsat 8.

\begin{tabular}{cccccc}
\hline DATA & HORÁRIO & $\mathbf{T}\left({ }^{\circ} \mathbf{C}\right)$ TORRE & T $\left(\mathbf{C}^{\circ}\right) \mathbf{B 1 0}$ & $\mathbf{T}\left(\mathbf{C}^{\circ}\right) \mathbf{B 1 1}$ & $\mathbf{T}^{\left(\mathbf{C}^{\circ}\right) \mathbf{B 1 0}-\mathbf{b 1 1}}$ \\
\hline $24 / 07 / 2016$ & $10: 00$ & 33,27 & 29 & 28 & 28 \\
\hline $10 / 08 / 2016$ & $10: 00$ & 25,39 & 23 & 22 & 22 \\
\hline $26 / 08 / 2016$ & $10: 00$ & 38,74 & 28 & 27 & 28 \\
\hline $11 / 09 / 2016$ & $10: 00$ & 37,83 & 31 & 28 & 29 \\
\hline $27 / 09 / 2016$ & $10: 00$ & 35,36 & 28 & 25 & 26 \\
\hline
\end{tabular}

O mapa de temperatura da superfície com um raio superior a $10 \mathrm{~km}$ da localização da torre 
(footprint) demonstra a espacialização dessa variável para cada dia de análise, apresentando valores que variaram de $15^{\circ} \mathrm{C}$ a $36^{\circ} \mathrm{C}$ (Figura 3 ). As mais altas temperaturas são constatadas em regiões de baixa vegetação, concentração de áreas urbanas e culturas, enquanto que as mais baixas temperaturas são detectadas nas áreas próximas aos corpos de água, rios e lagos (Figura 3).
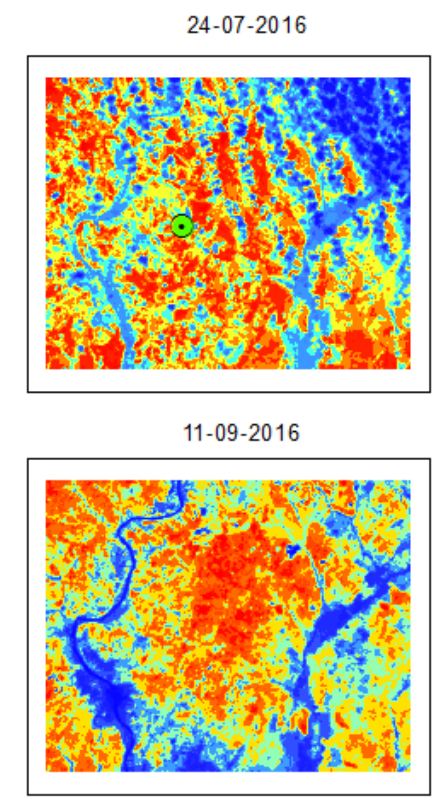

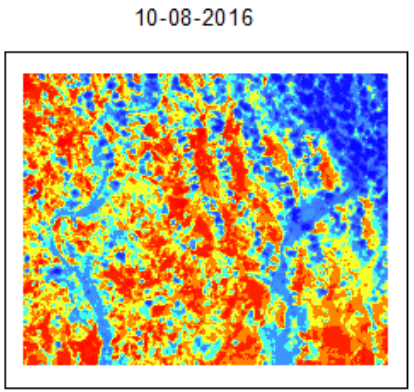

27-09-2016

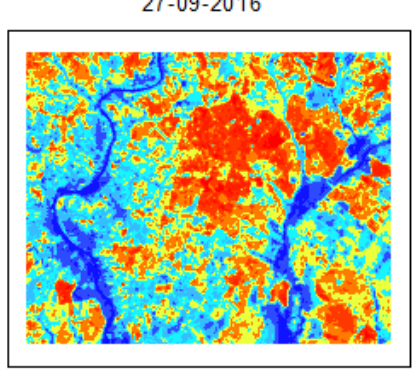

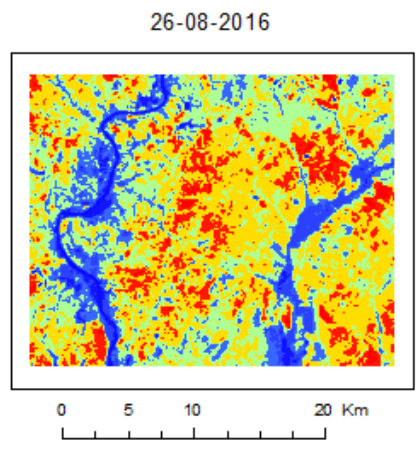

$\operatorname{LST}\left({ }^{\circ} \mathrm{C}\right)$

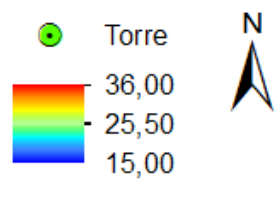

Figura 3. Mapa da temperatura da superfície.

No diagrama de caixa é possível analisar as diferenças das medianas encontradas nas cartas de temperatura da superfície. Durante todo período analisado, constata-se mediana de $28^{\circ} \mathrm{C}$ na área de estudo, demonstrando apenas uma diferença significativa no mês de agosto (Figura 4). Essa diferença é justificada pela mais baixa temperatura registrada (Tabela 1$)$.

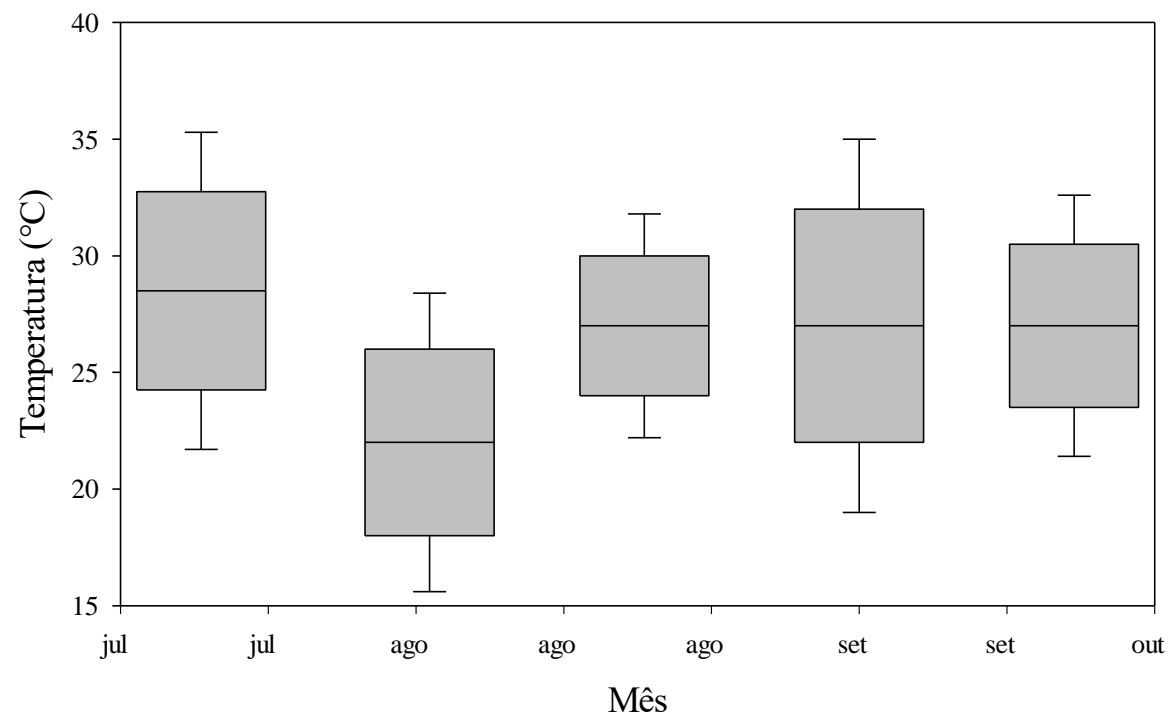

Figura 4. Diagrama de caixa da temperatura da superfície na área de estudo.

Quando extraído os dados de temperatura da superfície estimada pelo satélite Landsat $8 \mathrm{em}$ termos de frequência dos pixels (Figura 5), observa-se que as imagens que apresentaram maiores valores no pixel da torre (Tabela 1) 
demonstraram os intervalos de frequência distribuídos entre temperaturas mais elevadas em toda carta, de 22 a $32^{\circ} \mathrm{C}$, de forma que para as datas de imagens onde foram constatadas as mais baixas temperaturas no pixel da torre, os intervalos de frequência apresentaram temperaturas inferiores, de 15 a $30^{\circ} \mathrm{C}$ (Figura 5).

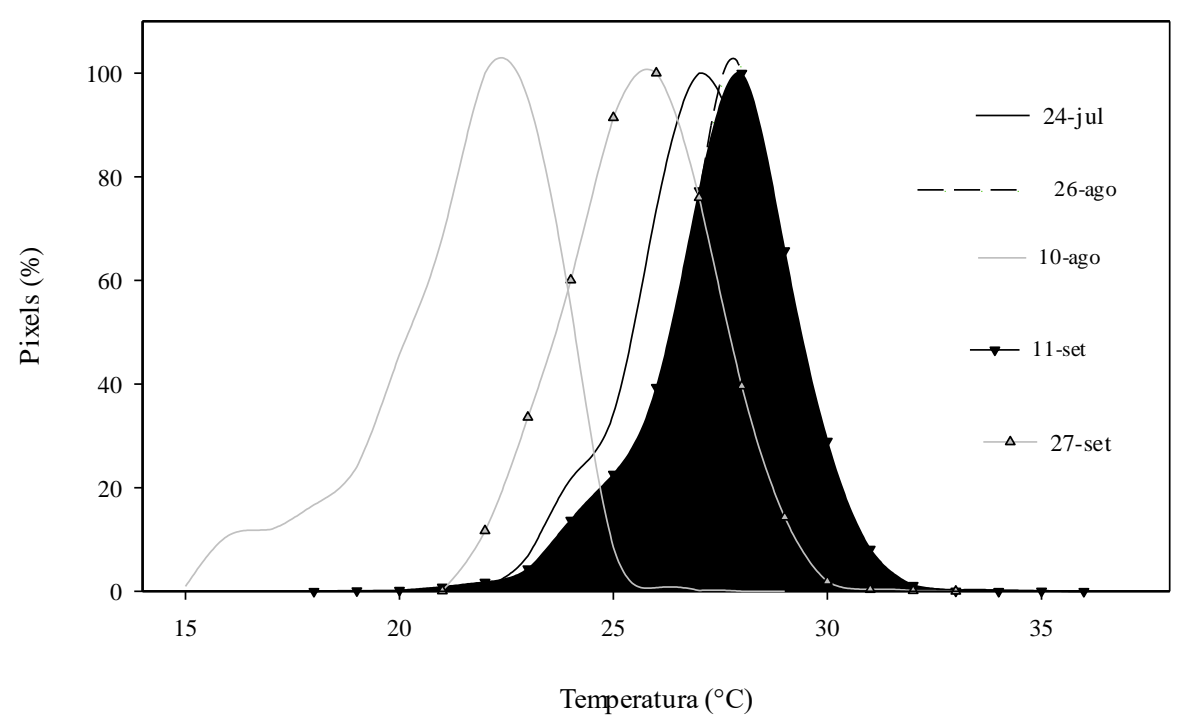

Figura 5. Histogramas das temperaturas da superfície na área de estudo nas datas correspondentes ao período de estudo: a) 24/07/2016, b) 10/08/2016, c) 26/08/2016, d) 11/09/2016 e e) 27/09/2016.

A análise parcial de cada banda termal do satélite Landsat 8 demonstra que a banda 10 do sensor TIRS apresentou valores mais elevados para a área de estudo quando comparada com a banda 11 (Tabela 1). Essas diferenças podem ser explicadas pelos diferentes intervalos de comprimento de onda de cada banda em resposta a interação com os alvos da superfície, de modo que, a banda 10 corresponde a uma faixa de 10,3 a 11,3 $\mu \mathrm{m}$, e a banda 11, de 11,5 a 12,5 $\mu \mathrm{m}$ (Barsi et al., 2014; Montanaro, et al., 2014).

Realizada a média de todas as imagens que correspondem a banda 10 e 11 constata-se maior mediana nas imagens que correspondem a banda $10,28,0^{\circ} \mathrm{C}$ e menor na banda 11 , de $25,8^{\circ} \mathrm{C}$, apresentando uma diferença de aproximadamente $2,2^{\circ} \mathrm{C}$ (Figura 6).

Afim de comparação, um mapa de diferença das bandas 10 e 11 foi gerado, destaca-se maiores diferenças nas regiões centrais da carta, onde foram encontradas as mais elevadas temperaturas, áreas essas que representam pouco ou ausência de vegetação, enquanto que as menores diferenças são constadas em áreas de corpos d'água (Figura 7).

Corpos d'água têm sido utilizados como alvos primários para calibrações das bandas termais dos satélites da série Landsat, justamente por possuir composição uniforme, apresentam conhecida e alta emissividade e exibe com frequência baixas variações de temperaturas (menores que $1{ }^{\circ} \mathrm{C}$ ) sobre todas áreas. Enquanto que, alvos terrestres apresentam as mais altas faixas de temperaturas e maior dificuldade de caracterização (BARSI et al., 2014; Knight \& Kvaran, 2014). 


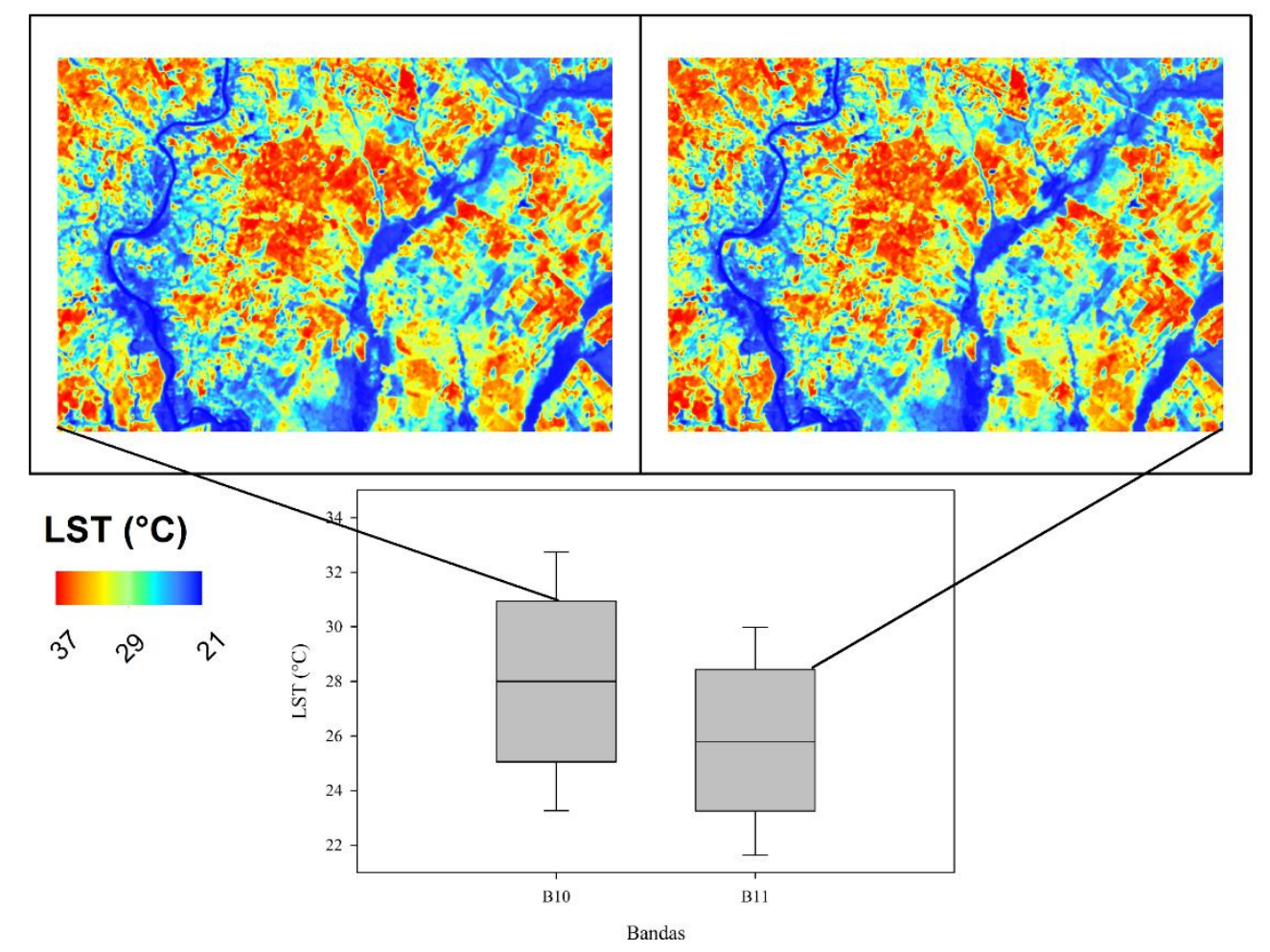

Figura 6. média das imagens que correspondem a banda 10, média das imagens que correspondem a banda 11 e o diagrama de caixa das medias das bandas 10 e 11 .

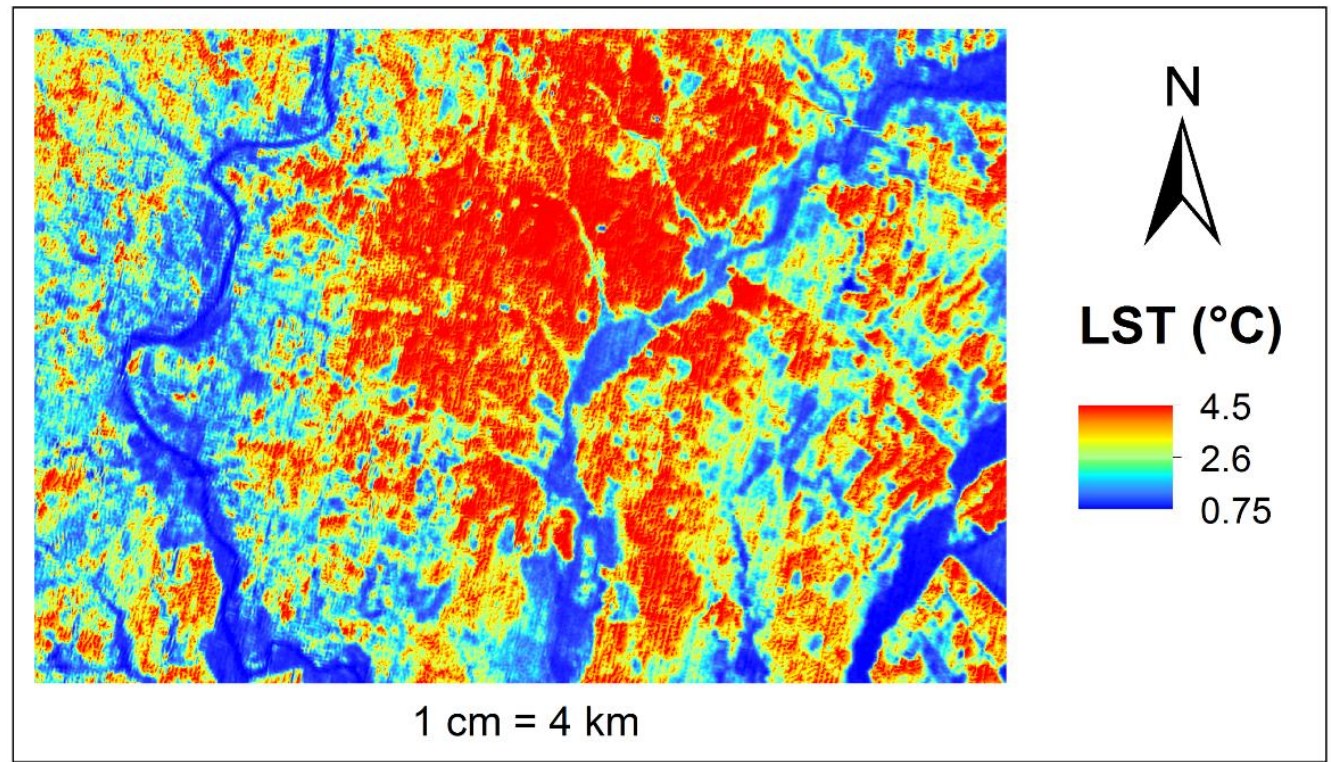

Figura 7. carta das diferenças de temperaturas das superfícies geradas a partir das imagens infravermelho termal (bandas 10 e 11) do Landsat 8.

Quando colocado em contraste a temperatura medida do ar medida em relação a estimada pelo satélite Landsat 8 , o índice de determinação $\left(R^{2}\right)$ e correlação de Pearson $(r)$ foram maiores com a média das duas bandas (bandas 10 e 11) termais do satélite (Figura 8). A banda 11 foi a que apresentou menores concordâncias com os dados medidos (Figura 5), possivelmente pelo fato de apresentar os menores valores para área de estudo, respectivamente (Tabela 1). 
Quando colocado em contraste as temperaturas medidas na torre e estimada pelo sensor TIRS do satélite Landsat 8 , a análise de variância (ANOVA) demonstrou que houve diferença significativa entre as médias das temperaturas medida e estimada $(p$-valor $=0,02)$.

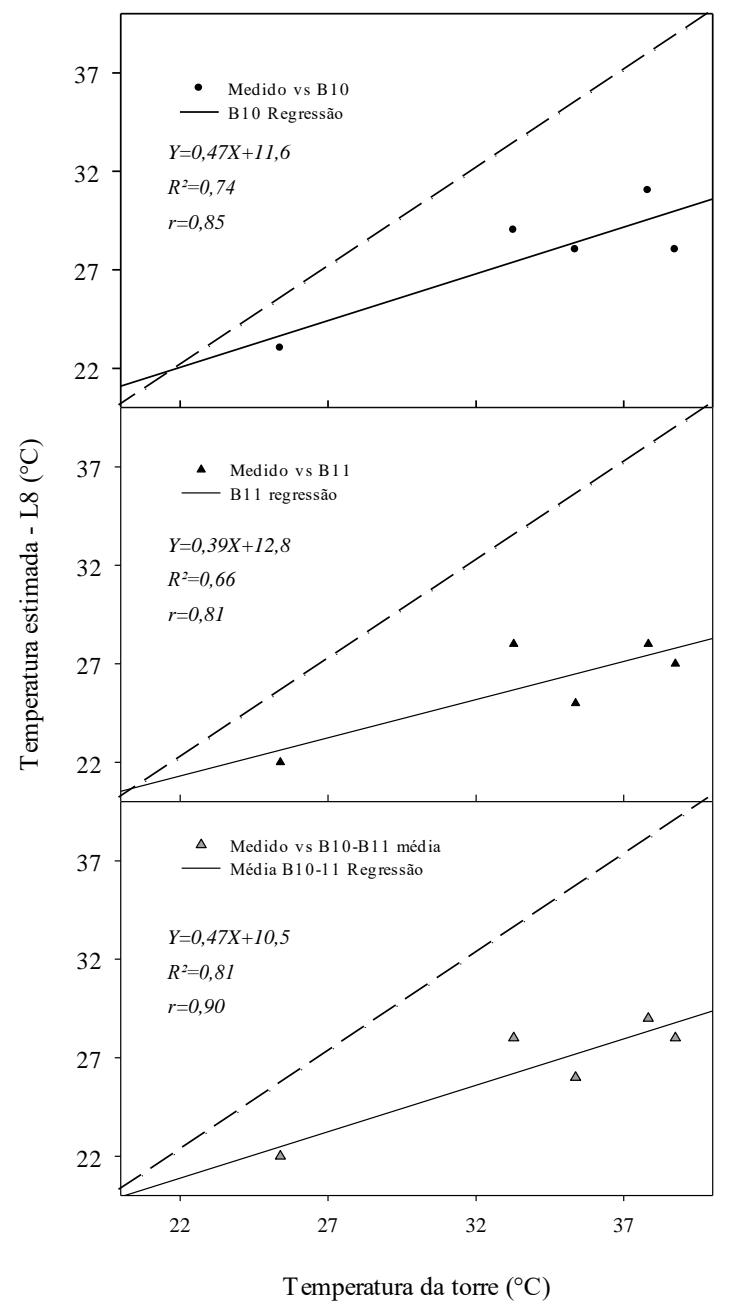

Figura 8. Regressões das temperaturas do ar medidas na torre com a temperatura da superfície estimada pelo satélite Landsat 8 (banda 10, Banda 11 e média das bandas 10 e 11).

\section{Conclusões}

Resultados encontrados nesse trabalho demonstraram o uso das bandas termais do satélite Landsat 8 no norte do Pantanal mato-grossense na discriminação de diferentes alvos na superfície. A análise parcial de cada banda do sensor TIRS (banda 10 e 11) do satélite Landsat 8 demonstrou valores mais elevados na banda 10 em comparação com a banda 11 .

A análise estatística dos dados de temperatura indica que as maiores correlações da temperatura medida na torre meteorológica foram constatadas com a média das bandas termais do satélite Landsat 8. A análise de variância demonstrou que houve diferença significativa entre as médias das temperaturas medida e estimada.

\section{Referências}

Alves, L. E., Gomes, H., Santos, M., Freitas, I. G. 2017. Balanço de radiação através do satélite Landsat-8 na bacia do Rio Pajeú. Revista Do Departamento De Geografia 33, 117-127.

Aquino, H. C., Galvanin, E. A. S., Neves, S. M. A., Lima, D, 2017. Análise da dinâmica de pastagem no Pantanal de Cáceres/MT. Geo UERJ, n. 30, 305-328.

Andrade, R. M., Bolfe, E. L., Victoria, D. C., Nogueira, S. F, 2017. Avaliação das condições de Pastagens no Cerrado por meio de 
Geotecnologias. Revista Brasileira de Agropecuária Sustentável (RBAS) 7, 34-41.

Anjos, A. W., Delgado, R. C., Oliveira Junior, J. F., Gois, G., Moraes, N. O., 2013. Temperatura da superfície continental associada aos eventos meteorológicos na cidade do Rio de Janeiro, RJ. Enciclopédia Biosfera 9, 3692-3707.

Barsi, J. A., Schott, J. R., Hook, S. J., Raqueno, N. G., Markham, B. L., Radocinsk, R. G., 2014. Landsat-8 Thermal Infrared Sensor (TIRS) Vicarious Radiometric Calibration. Remote Sensing 6, 11607-11626.

Cristóbal, J., Muños, J. C. J., Prakash, A., Mattar, C., Skokovic, D., Sobrino, J., 2018. An Improved Single-Channel Method to Retrieve Land Surface Temperature from the Landsat- 8 Thermal Band. Remote Sens. 10, 2-14.

Dallacort, R., Neves, S. M.A. S., Nunes, M. C. M., 2014. Variabilidade da temperatura e das chuvas de Cáceres/Pantanal Mato-Grossense. Geografia 23, 21-33.

Danelichen, V. H. M., Biudes, M. S.; Souza, M. C., Machado N. G., Silva, B. B., Nogueira, J. S., 2014. Estimation of soil heat flux in a Neotropical Wetland region using remote sensing techniques. Revista Brasileira de Meteorologia 29, 469 - 482.

Danelichen, V. H. M., Biudes, M. S.; Souza, M. C., Machado N. G., Nogueira, J. S., 2014. Relations of vegetation and water indices to volumetric soil water content in the Pantanal of Mato Grosso, Brazil. Journal International Journal of Remote Sensing 37, 4261-4275.

Knight, E.; Kvaran, G., 2014. Landsat-8 Operational Land Imager Design, Characterization and Performance. Remote Sens, vol. 6, p. 10286-10305.

Knox, S. H., Dronova, I., Sturtevant, C., 2017. Using digital camera and Landsat imagery with eddy covariance data to model gross primary production in restored wetlands. Agric. For. Meteorol. 237, 233-245.

Lima, S., Barrozo, L., Mataveli, G., 2018. Temperatura da Superfície e Precipitação que Influenciam na Incidência do Aedes Aegypti em São Paulo. Revista Do Departamento De Geografia 174-183.

Miranda, M. R. S., Luz, C. C. S., Neves, S. M. A. S., Scheuer, J. M, 2018. Cobertura vegetal e uso da terra no Assentamento Paiol-Brasil: Subsídios Para a Conservação do Pantanal. Revista de Geografia - PPGEO - UFJF 8, 5968.

Montanaro, M., Levy, R., Markham, B., 2014. OnOrbit Radiometric Performance of the Landsat
8 Thermal Infrared Sensor. Remote Sens 6, 1753-11769.

Santos, F. A. C., Santos, A. C. S., Silva, B. B., Araújo, A. L., Cunha, J. E. B. L., 2015. Desempenho de Metodologias para estimativa do Saldo de Radiação a Partir de imagens Modis. Revista Brasileira de Meteorologia 30, 295-306.

Santos, M. M., Machado, I. E., Carvalho, E. V., Viola, M. R., Giongo, M., 2017. Estimativa de Prâmetros Florestais em área de Cerrado a partir de imagens do sensor OLI Landsat 8. FLORESTA 47, 75-83.

Silva Junior, C. H., Anderson, L., Aragão, L. E., Rodrigues, B., 2018. Dinâmica das Queimadas no Cerrado do Estado do Maranhão, Nordeste do Brasil. Revista Do Departamento De Geografia 35, 1-14.

Souza, M. C., Biudes, M. S., Danelichen, V. H. M.; Machado, N. G., Musis, C. R.; Vourlitis, G. L., Nogueira, J. S., 2014. Estimation of gross primary production of the Amazon-Cerrado Transitional Forest by remote sensing techniques. Revista Brasileira de Meteorologia 29, 1-12.

Souza, C. A., Danelichen, V. H. M., 2018. Uso de Imagens de Satélite Como Ferramenta para Análise da Vegetação do Monumento Natural Morro de Santo Antônio/MT. UNICIÊNCIAS, 22, 17-19.

Souza, C. A., Danelichen, V. H. M., Biudes, M. S., Silveira, W. G., 2018. O uso de Sensoriamento Remoto para estimar o parâmetro Florestal de Unidade De Conservação em Área de Cerrado. Revista Brasileira de Geografia Física, v. 11, n. 5, p.1-12.

Marques, H. O., Biudes, M. S., Pavão, V. M., Machado, N. G., Querino, C. A. S., Danelichen, V. H. M., 2017. Estimated net radiation in na Amazon-Cerrado transition forest by Landsat 5 TM. J. Appl. Remote Sens. 11, 2-11.

Masiello, G., Serio, C., Venafra, S., Liuzzi, G., Göttsche, F., F. Trigo, I., Watts, P., 2015. Kalman filter physical retrieval of surface emissivity and temperature from SEVIRI infrared channels: A validation and intercomparison study. Atmos. Meas. Tech. 8, 2981-2997.

Muniz, R. A., Vettotazzi, C. A., Martorano, L. G., Dias, C. T. S., Lisboa, L. S. S, 2016. Dinâmica Espaço-Temporal Da Temperatura De Superfície, Da Bacia Do Rio Corumbataí, SP Utilizando Imagens Do Sensor Tm/Landsat. Revista Engenharia na Agricultura 31, 169-177.

Rosas, J., Houborg, R., McCabe, M. F., 2017. Sensitivity of Landsat 8 Surface Temperature 
Estimates to Atmospheric Profile Data: A Study Using MODTRAN in Dryland Irrigated Systems. Remote Sens. 9, 4-27.

Strassburge, A. S, Menezes, A. J. E. A., Perleberg, T. D., Eicholz, E. D., Mendez, M. E. G., Schöffel, E. R., 2011. Comparação da temperatura do ar obtida por estação meteorológica convencional e automática. Revista Brasileira de Meteorologia 26, 273 278.

Takatori, M. M. O., Gamarra, R. B., Faggioni, G. P., Dalmas, F. B., Paranhos Filho, A. C, 2017. Uso de Geotecnologias na análise da estrutura e dinâmica da Paisagem na região do Nabiquele. Revista UNG - Geociências 16, 87-101.

Velasque, M. C. S., Biudes, M. S., Machado, N. G., Danelichen, V. H. M., Vourlitis, G. L., Nogueira, J. S., 2018. Modelling Gross Primary Production of Tropical Forest by Remote Sensing. Revista Brasileira de Climatologia 22, 38-54.

Vigano, H. H. G., Souza, C. C., Cristaldo, M. F., Neto, J. R. N., Jesus, L, 2018. Incêndios no Pantanal de Corumbá, MS: modelagem e previsão a partir das técnicas de análise multivariada. Rev. Ambient. Água 13, 1-13.

Vilani, M. T., Reis, N. M. S., Pinto Junior, O. B., Novais, J. W. Z., 2018. Coerência Wavelets em Variável Temperatura do Ar em Área do Bioma Pantaneiro e Área de Alta Densidade Construtiva. Ensaios Cienc 22, 5-11.

Vermote, E., Justice, C., Claverie, M., Franch, B., 2016. Preliminary analysis of the performance of the Landsat 8/OLI land surface reflectance product. Remote Sens. Environ. 185, 46-56.

Zhou, Y., Xiao, X., Qin, Y., Dong, J., Zhang, G., Kou, W., Jin, C., Wang, J., LI, X., 2016. Mapping paddy rice planting area in ricewetland coexistent areas through analysis of Landsat 8 OLI and MODIS images. Remote Sensing of Environment. 46, 142-154.

Zibognon, M., Rago, R., S Uleiman, A., 2002. Conversion of radiometric to aerodynamic surface temperature with an anisothermal canopy model. Resources Research. Water Resources Research 38, 1067. 